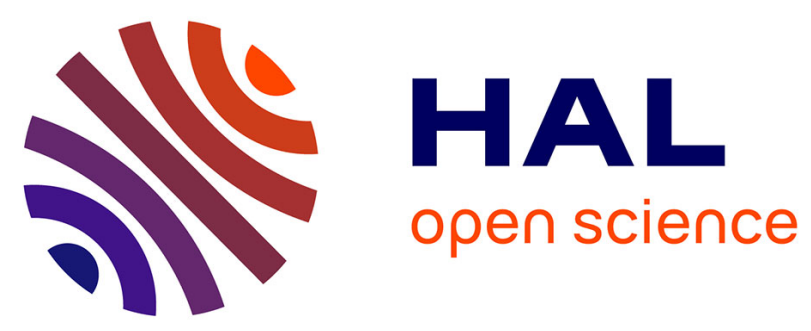

\title{
Visual Measurements at Small Scales: Guidelines to Reduce Uncertainties down to a Few Nanometers
}

\author{
Benjamin Mauze, Redwan Dahmouche, Cédric Clevy, Patrick Sandoz,
} François Hennebelle, Guillaume Laurent

\section{To cite this version:}

Benjamin Mauze, Redwan Dahmouche, Cédric Clevy, Patrick Sandoz, François Hennebelle, et al.. Visual Measurements at Small Scales: Guidelines to Reduce Uncertainties down to a Few Nanometers. International Conference on Manipulation, Automation and Robotics at Small Scales (MARSS 2020), Jul 2020, Toronto, Italy. 10.1109/MARSS49294.2020.9307854 hal-03186546

\section{HAL Id: hal-03186546 https://hal.science/hal-03186546}

Submitted on 31 Mar 2021

HAL is a multi-disciplinary open access archive for the deposit and dissemination of scientific research documents, whether they are published or not. The documents may come from teaching and research institutions in France or abroad, or from public or private research centers.
L'archive ouverte pluridisciplinaire HAL, est destinée au dépôt et à la diffusion de documents scientifiques de niveau recherche, publiés ou non, émanant des établissements d'enseignement et de recherche français ou étrangers, des laboratoires publics ou privés. 


\title{
Visual Measurements at Small Scales: Guidelines to Reduce Uncertainties down to a Few Nanometers*
}

\author{
Benjamin Mauzé ${ }^{1}$, Redwan Dahmouche ${ }^{1}$, Cédric Clévy ${ }^{1}$, Patrick Sandoz ${ }^{1}$, \\ François Hennebelle ${ }^{2}$ and Guillaume J. Laurent ${ }^{1}$
}

\begin{abstract}
Vision-based measurement is a widespread solution to characterize, classify and control micro and nano robotic systems. Achieving multi-degrees-of-freedom measurements with nanometer uncertainties using a common experimental setup has recently been demonstrated which appears highly interesting for many applications. Nevertheless, succeeding in setting the experimental set-up to reproduce such performances still appears not so easy, due to the difficulty to understand what are the influence quantities. To address this key issue, the paper firstly synthesizes the different approaches and good practices used in the literature where several influence quantities have mainly been investigated independently. Secondly, an experimental set-up is used to study the influence of several key quantities onto position uncertainties. These two kinds of investigations, conducted to propose practical guidelines to get position uncertainties down to $5 \mathrm{~nm}$ with typical experimental setups used for micro and nano robotics purposes. The experiments also show that measurement precision is experimentally estimated at a few nanometers by measurement reproducibility over several days.
\end{abstract}

\section{INTRODUCTION}

For two decades, activities at micro and nano scales are multiplying. Many prospects and applications have been identified in research and industry such as for optics, microelectronics, nanomaterials, biology, chemistry, etc [1]. To meet the increasing needs of accurate positioning in robotics, the first key lock lies in a measurement systems able to reach nanometer precision. For instance, Popa et al [2] designed a multiscale robot platform and pointed out the importance of the sensor choice that can become a limiting element. More generally, sensing at very small scale is particularly challenging in terms of integration, range-to-precision ratio and sensibility to perturbations. Liaw and Shirinzadeh [3], in their impedance control application, turned down the use of a laser-interferometry-based sensor for a capacitive one because of the lack of space to put the retro-reflector. Wang et Zhang [4] highlighted the lack of multidimensional sensors by using three capacitive sensors in order to measure the displacement and the orientation of their nanopositioning platform.

\footnotetext{
*This work was supported in part by the French Investissements d'Aveni program ISITE-BFC (contract ANR-15-IDEX-03), by Equipex ROBOTEX (ANR-10-EQPX-44-01), by Région Bourgogne Franche-Comté and the EIPHI Graduate School (ANR-17-EURE-0002).

${ }^{1}$ B. Mauzé, R. Dahmouche, C. Clévy, P. Sandoz and G. J. Laurent are with the FEMTO-ST Institute, Univ. Bourgogne FrancheComté, UMR CNRS 6174, Besançon, France. Corresponding author: cedric.clevy@femto-st.fr

${ }^{2}$ F. Hennebelle is with DRIVE EA1859, Univ. Bourgogne FrancheComté, Nevers, France.
}

Another widespread solution is vision. Feng et al [5] highlighted significant advantages of vision-based sensors as their ability to achieve multi-degrees-of-freedom measurements and their ease of implementation even at small scales. Compared to other sensors, vision-based measurement setups recently shown consistent results. For example, resolutions below one nanometer have been experimentally demonstrated with a visual position measurement method that uses a regular camera, a microscope and pseudo-periodic patterns [6], [7]. In this scope, vision appears particularly relevant as measurement to get nanometer position uncertainty.

But, if vision-based measurement is seen as a rising trend, quantifying the uncertainties is a key for many works [8]. At the micro scale, this evaluation appears all the more difficult than at the macro scale due to the complexity of revealing what are the influence quantities. Besides, for the measurement processes conventionally carried out in microrobotics, there is no complete assessment of measurement uncertainties.

At the macro scale, several key guidelines and standards exist. They all mention, as preliminary hypothesis, the necessity to fix any quantities that may induce drift to successfully achieve exploitable experimental measurements (measurement standards such as ISO GUM [9]) or to achieve low position uncertainties (ISO 9283:1998 standard [?]). This hypothesis appears especially strong at the micro and nano scales because many quantities induce time dependant behaviors such as drifts that directly and strongly induce large position errors [10]. Moreover, these biases often result from several influence quantities that act together. Identifying the most influential ones and being able to quantify the relative influence of every parameter thus appears to be a key challenge.

This paper deals with the establishment of practical guidelines for typical vision-based micro and nano robotics setups by understanding what are the main sources of uncertainties. Beyond previous studies that focus on the impact of one of few uncertainty factors at a time, in this paper aims at defining practical guidelines to reach position measurement errors down to a few nanometers. To do so, related works investigating sources of uncertainty in computer vision are firstly analysed (Section II). Then, a generic experimental measurement setup with a microscope and a camera is used. Several experimentations are conducted to clearly understand the main influence quantities (Section III). Those experiments are conducted to demonstrate that a nanometer 


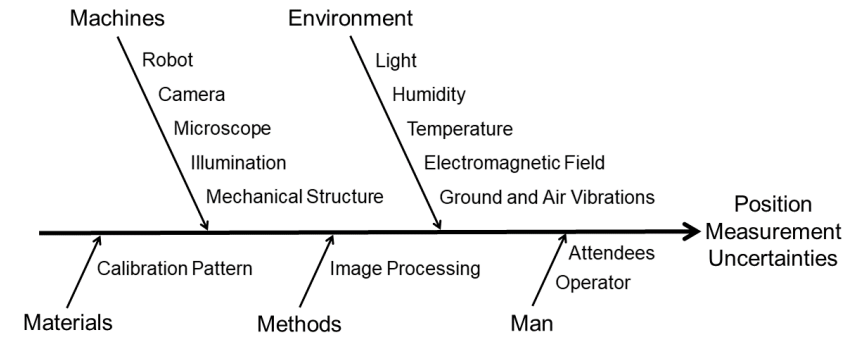

Fig. 1: Ishikawa diagram identifying potential sources causing position measurement uncertainties at micro and nano scales.

position uncertainty can be obtained, that is also confirmed by the reproducibility of the results over a long period of time (Section IV).

\section{LITERATURE ANALYSIS OF INFLUENCE QUANTITIES AT SMALL SCALES}

A measurand must be expressed as a quantity value and its associated uncertainties, which correspond to the dispersion which can be attribute to the quantity characterized. At micro and nano scales, the signal-to-noise ratio can be poor even for physical parameters such as position measurement. Identifying, quantifying and canceling most influence quantities on the assessment uncertainties is essential to obtain accurate measurements.

From literature and common knowledge, several potential sources of uncertainty have been identified. They depend on the experimental setup, on human interactions, on environmental conditions, and on the measurement method itself (image processing and calibration pattern). Fig. 1 shows an Ishikawa diagram which identify the main influence quantities on the position measurement (measurand evaluated) assessment. In the following, these sources are detailed for typical microrobotic setups relying on visual measurements.

\section{A. Machines}

Camera: The resolutions of the used cameras have a direct impact on position measurement quality [11]. However, exposure time, electronic and numerical corrections, like gamma gain that enhances the contrast by applying a nonlinear intensity scale, also have an influence on the quality of the measurement because of associated image distortions, noise or bad contrasts. For instance, Pan et al [12] pointed out the importance of image quality for digital image correlation methods. Also, Ho [13] showed that digitizing, low contrast and low brightness have an impact on the measurement result.

Results may also depend on the type of the sensor (CDD or CMOS). Historically, CDD sensors were known to be less noisy but can be affected by pixel jittering [14].

Microscope: The choice of an appropriate microscope objective to obtain the desired image resolution and field of view is mandatory [15]. Objective lenses with large numerical aperture improve the optical resolution but reduce the depth of field and the working distance. A balance has to be found between resolution and depth of field.
From the geometrical point of view, measuring displacements perpendicular to the optical axis reduces uncertainties [16]. In this case, geometrical disfigurements and defocus effects are reduced.

Mechanical structure: To get the most stable structure that holds the camera and the microscope, the stand must be stiff and heavy enough to avoid mechanical disturbances. Shirmohammadi and Ferrerro [8] underlined that the vibrations from the environment, the camera stand's imperfections and the induced angle are potential sources of measurement errors. In metrology applications, granite is generally used to add weight while it is not the case for most of microrobotic setups. Besides, all structural elements need to be in the experimental room for a long time (more than $24 \mathrm{~h}$ ) before performing the experiments, otherwise temperature gradient might induce drifts.

Illumination: Illumination conditions should be correctly tuned before starting the acquisitions. Indeed, the impact of illumination intensity and position studied in [17] demonstrated that a good illumination must show as much contrast as possible without introducing shadows. Warmth absorbed by dark samples can induce drifts. Depending on the material and the experiment condition, backlight can be considered.

Increasing the illumination power may allow an electronic gain reduction with subsequent noise mitigation. Caution must however be taken to avoid illumination power fluctuations and camera saturation. Depending on the sample, adjustment of the illumination angle of incidence may lead to an increased contrast, for instance by differentiating more efficiently between reflective and scattering zones of a textured target. In the case of multi-material targets, the adjustment of the mean illumination wavelength to the materials' spectral reflectivities may also improve the image contrast.

In addition, thermal convection and drift induced by lighting can cause measurement errors. Using cold sources can avoid thermal problem and provide stable light sources reducing the uncertainties.

\section{B. Method - Image processing}

Many image processing methods have been reported to measure displacements of microrobots. Feature-based [18] and area-based methods [19] have been both used. Last ones are preferred at small scales because of their high resolution and robustness to noises. Among them, it has been demonstrated that photometric methods like image correlation can achieve nanometer resolution using regular 20x microscopes [20]. Direct phase measurements can even reach sub-nanometer resolution and are even more robust to noise, to blur and to partial occlusions. These methods rely on phase calculated by the Fourier transform of the image [21], [22]. They can be used together with calibrated periodic patterns gauge to get accurate measurements of positions [7].

\section{Environment}

Temperature: Temperature is a very influence quantity. For instance, Tan et al [23] experimentally showed that drift can reach $1.4 \mu \mathrm{m}$ for $1^{\circ} \mathrm{C}$ thermal variation with a 
typical microrobotic setup. Thermal variations are usually unpredictable and highly complex to model since they are induced by various sources (e.g. people, electronics, lighting and internal temperature of the elements of the setup) [24]. Doing experiments within a thermally regulated room and increasing thermal inertia of the setup surroundings can prevent big drifts.

Humidity: Humidity is known to be very problematic because of its effects on friction and contact, especially in the case of microrobotic assembly tasks [25]. However, its impact on measurement quality seems to remain very small. Nevertheless, some precautions such as using a dehumidifier and monitoring humidity variations can be taken.

Electronic, electromagnetic fields, electrostatic: Meng and Zhuang [26] pointed out electronic noises (a.e. jitter) as a main source of reducing the precision of the image. Other sources like electromagnetic fields or electrostatic forces can disturb the measurement. Depending on the applications, those sources need to be considered with a specific attention. Even if their influences are low on optical waves involved in visual measurements, regulated power supplies has to be considered notably for the illumination sources.

Ground and acoustic vibrations: All mechanical structures are sensitive to vibrations especially at small scales. To reduce the propagation of ground vibrations, tables with pneumatic isolation systems are mandatory.

Cantilever structures have also to be avoided because of vibrations. De Faria et al [27] demonstrated the influence of mechanical noises on components inside a scanning electron microscope. They showed that, depending on the frequency, mechanical vibration noises can induce errors from $1 \mathrm{~nm}$ to more than $20 \mathrm{~nm}$ at the tip of an atomic force microscope. In ambient environment, air flows and connection wires may also introduce disturbances which causes measurement errors.

Ambient light: Intensity fluctuations from sunlight or artificial light (fluorescent lamp for instance) can affect the image processing output. It is recommended to build a black box around the experimental setup and hide the windows with special reflective film. It is also interesting to be able to work in a room not subject to sun light variations (always in the shade for example or in the basement).

\section{Man - Human attendance and work}

Human presence during experiments at very small scale is a real problem. For example, a person induces heat and breath upon the manipulation resulting into unwanted vibrations and variations of the temperature. Moreover, potential ground vibrations impacting the experiment increase as well as the possibility of inadvertent moving. Errors increase with the number of people which are near the experimental setup.

Operators changing can also have an influence. Thus it is important to write a measurement procedure, which must be as complete as possible, to reduce the human influence. Tightenings must for example be carried out in the same order and with a torque wrench.

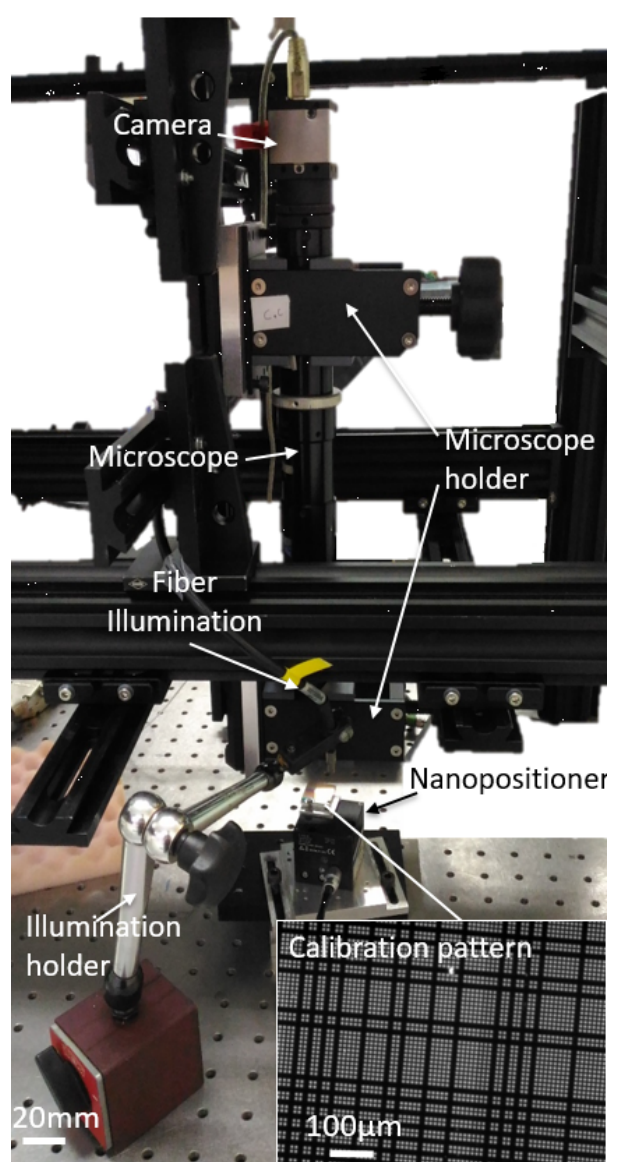

Fig. 2: Experimental setup consisting in a camera, its holder and pattern.

Thus, automating the measurement increases its reproducibility and reduces the risks of human interactions.

\section{E. Materials - Calibration pattern}

Calibrating the camera thanks to a pattern gauge is a common procedure to get a better accuracy. The patterns themselves have some defaults due to their fabrication process. Nevertheless, the dedicated machines are regularly calibrated and installed in clean room with strict fabrication protocols. Thus, the fabrication default of the patterns are generally small and known from certified characterization tests performed by authorised laboratories like NIST or LNE.

The phenomena related to the mechanical structure, the ground and acoustic vibrations and human attendance might also occur in other microscopy types such as Scanning electron microscopes, Atomic force microscopes and Scanning tunnelling microscopes.

\section{EXPERIMENTAL EVALUATION OF VISUAL MEASUREMENT UNCERTAINTY}

\section{A. Experimental Setup}

All the experiments have been performed with the same microrobotic architecture. Fig. 2 shows the setup used for this measurement campaign and is detailed below. All guidelines presented in the previous section have been considered in order to get the best measures. 


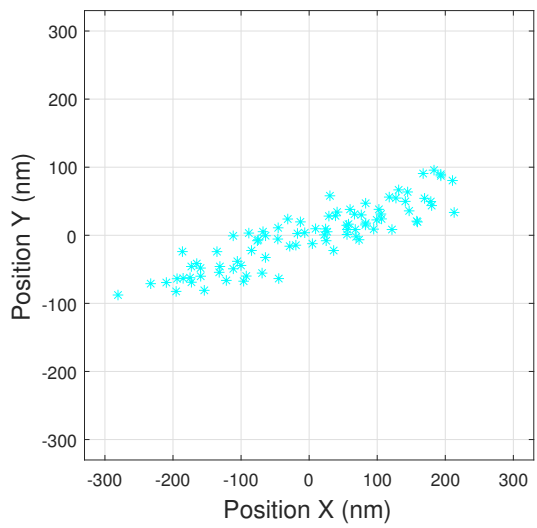

(a)

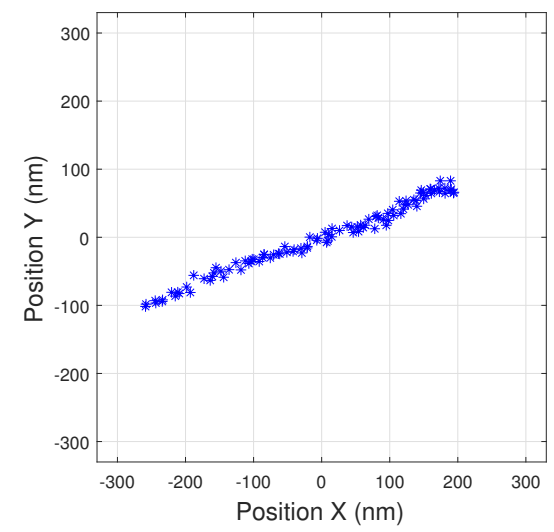

(b)

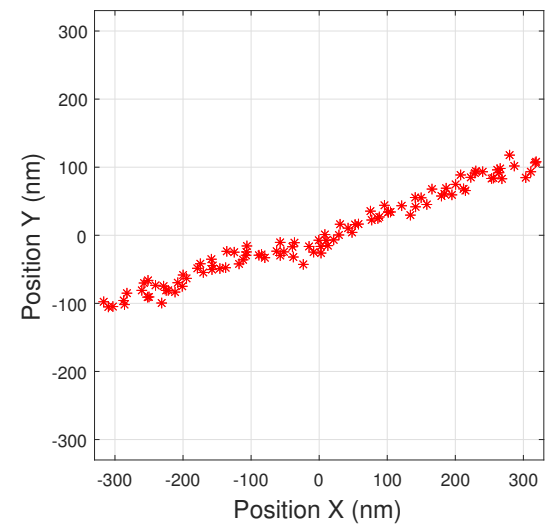

(c)

Fig. 3: Evolution of the position of the calibration pattern in the camera frame over time considering different experimental conditions. The duration of each experiment is 90 seconds. (a) Position just after the assembly and lighting warm-up. Maximum distance to mean: $294 \mathrm{~nm}$. (b) Position after waiting for mechanical relaxation and lighting warm-up. Maximum distance to mean: $278 \mathrm{~nm}$. (c) Position after waiting for mechanical relaxation but without lighting warm-up. Maximum distance to mean: $337 \mathrm{~nm}$.

This first setup (named "Setup \#1") is composed of an IDS UI-2230SE-M-GL CCD camera $(1024 \times 768$ pixel array, 8 bits, exposure time $30 \mathrm{~ms}$ ) used at 15 frames per second with an optical microscope and a $10 \times$ Mitutoyo WD PLAN APO objective lens (numerical aperture 0.28).

The camera is focused on a calibration pattern gauge positioned on a static surface or on a nanopositioner at an idle position (NanoCube ${ }^{\circledR}$ P-611-3S from Physik Instrumente). The calibration pattern was manufactured by etching a chromium layer onto a transparent glass wafer. The periodicity between points is $10 \mu \mathrm{m}$ along the two directions. The pattern used was fabricated by direct laser writing with a commercial instrument (Heidelberg DWL200) whose position and displacements are continuously controlled by a $\mathrm{HeNe}$ laser interferometer. Front lighting is provided by a cold light source (Edmund optic MI-150) and fiber optic to leverage the thermal impact of the light on the viewed scene. In addition, an antivibration table is also used to mitigate the impact of ground vibrations on the setup from ambient vibrations. All elements of the setup were in the room for a long period in order to reach thermal equilibrium, while the experimental room is under thermal regulation $\pm 1^{\circ}$. All screws were firmly tightened to have a stable and rigid mechanical structure.

Images and time are recorded with $\mathrm{C}++$ software. Both software run under Windows 7 on a PC: Intel Xeon CPU $51620 \mathrm{v} 33.5 \mathrm{GHz}$.

To measure the visual pattern position, the camera records more than 20 spatial periods in order to get the most precise measurements. Image processing is performed offline and relies on direct phase measurement as described in [22], [28]. It provides the $\mathrm{X}$ and $\mathrm{Y}$ positions of the center of the image in the pattern frame with a sub-nanometric resolution as well as rotational angle. Time is recorded at the same time as the images. Except for the last presented experiment, all the other ones last 90 seconds (which is chosen as a trade-off duration between long enough duration to highlight potential stability of the experimental setup and statistically representative enough sets of measurement data).

\section{B. First Results}

Since the previous guidelines were considered, the experiments should allow to characterize the best possible uncertainty on the positioning of the robot. Nevertheless, as it can be seen in Fig. 3a, positions dispersion is not Gaussian and is subject to a drift: unwanted displacements reached $493 \mathrm{~nm}$ in the $X$ direction and about $184 \mathrm{~nm}$ in the $\mathrm{Y}$ direction during the 90 seconds recordings. Maximum distance to the mean is $294 \mathrm{~nm}$. Such a high error observed in less than two minutes is an obstacle to micro and nanorobotic applications.

The nanopositioner was removed and the calibration pattern was put on honeycomb optical breadboard to eliminate the possibility that errors were coming from holder position displacements. Results were in the same order of magnitude. Thus, additional influence quantities have been investigated in the following.

\section{ADDITIONAL INFLUENCE QUANTITIES}

\section{A. Mechanical relaxation}

As the first experiment was conducted just after mechanical adjustments, the hypothesis of one source is the relaxation of stress constraints. In order to confirm it, another experiment was performed with the same experimental conditions but after waiting more than one day after tightening all the screws.

Results are shown in Fig. 3b. An important drift is still present but the dispersion is smaller than before. In fact, the range of unwanted displacements is still of $278 \mathrm{~nm}$. 


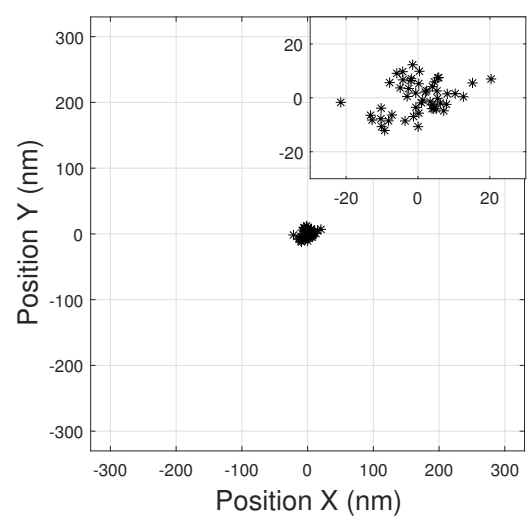

Fig. 4: Final experimental results following all the proposed guidelines (notably waiting for mechanical relaxation, lighting and camera warm-up). Maximum distance to mean: $22 \mathrm{~nm}$. Std: $5 \mathrm{~nm}$.

\section{B. Lighting warm-up}

Among other potential sources, lighting warm-up has been tested and quantified. The illumination source has been switched on just before starting the experience. Its influence upon thermal fluctuations or the processing algorithm can be shown by the increase of the maximum distance to mean which reaches $337 \mathrm{~nm}$ (Fig. 3c).

\section{Camera warm-up}

Numerous experiments were performed with different experimental conditions but errors remained still high. In order to see if the drift remains with time, a day-long experiment was performed and we observed that the drift was reducing with time. One can assume that it is caused by thermal dilation of the camera which could need a more appropriate warm-up phase. To prove it, another experiment was accomplished with an appropriate warm-up cycle. Of course camera was always switched on a long time (more than several hours) before experiments but not in grabbing mode. A more effective camera warm-up consists in running the camera around 1 hour at experimental conditions (i.e. in grabbing mode with same frame rate), before starting recording images. The results with this camera warm-up is shown in Fig. 4. The maximum distance to mean is reduced to $22 \mathrm{~nm}$. The improvement is more than a factor 10 .

This results can be related with the work of Handel [29]. He analyzed the influence of camera warm-up on image acquisition at macro scale for 45 minutes experiments. His results are given in pixels and the shapes of the drifts are similar to ours. Considering the duration of our experiment, for the top left target, he observed 0.08 and 0.04 pixels in $\mathrm{X}$ and $\mathrm{Y}$ directions while we got 0.09 and 0.04 . Handel proposed a model to compensate for this thermal effect but the method requires a temperature sensor within the camera. For microrobotic purposes, a long camera warm-up is easier to implement and can be applied directly to all devices.

\section{REPRODUCIBILITY}

In order to investigate the reproducibility of the results over a longer period of time, two additional tests have been

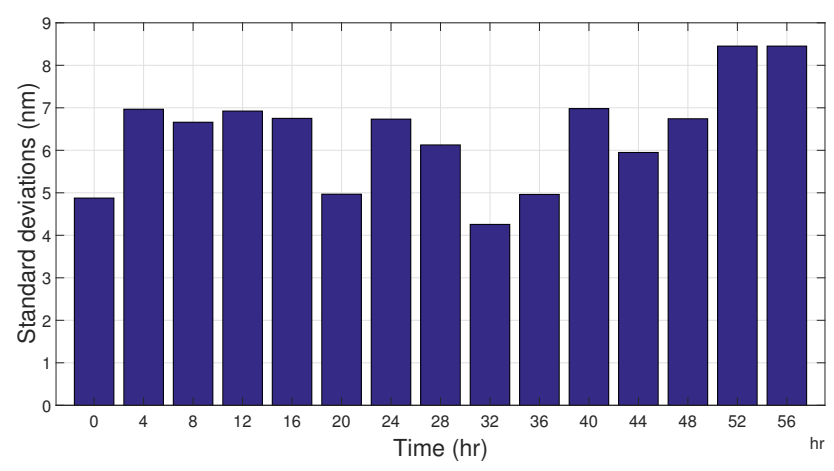

Fig. 5: Standard deviations of displacements during long periods (56 hours) showing the high stability of the measurement process.

carried out. The first one consists in taking series of images during 90 seconds every four hours during more than two days ( 56 hours). Actions have been established to be sure that nobody will interfere with the experiment.

The obtained results are presented in Fig. 5. The standard deviation of the errors are in the same order of magnitude of the one shown in the previous section. Indeed, one can see that none of the standard deviations are higher than $9 \mathrm{~nm}$. The reproducibility standard deviation, defined as the root square of the mean of error variances, is $4 \mathrm{~nm}$.

The second test that has been carried out to investigate the reproducibility of the results over a long period of time, consist to study the equipment-dependency. To do so, additional experiments were performed in similar conditions but with an IDS UI-3880CP-C-HQ CMOS camera (3088*2076 pixel array, 8 bits) and another Optem microscope tube, this setup is named "setup \#2". Results are similar to those presented in Fig. 4. Standard deviation is also equal to $5 \mathrm{~nm}$ and maximum distance to mean is about $24 \mathrm{~nm}$. This demonstrates that the proposed guidelines can be used for common setups without any specific action. This points out the good reproducibility of this vision-based system to perform measurements in the nanometer range when following all the established guidelines.

\section{CONCLUSION}

Performing tasks at micro and nano scales, such as manipulation and characterization, is often limited by measurement capabilities caused by numerous disturbance sources. In this article, those sources have been studied based on both stateof-the-art practices used and experimental investigation considering common vision-based measurement architectures used in micro and nanorobotics.

Tab. I summarizes the effect of the experimented sources of uncertainty. As between two consecutive lines only one factor is changing, we can calculate the relative improvement of a action on the maximum error, giving an order of magnitude of their impact for the $90 \mathrm{~s}$ duration of the experiment. Lighting warm-up reduces the maximal error from $337 \mathrm{~nm}$ to $278 \mathrm{~nm}$ corresponding to a relative decrease of $17 \%$. Waiting for mechanical stability reduces the maximal error by $16 \mathrm{~nm}$, corresponding to a variation of $5 \%$. The most predominant 


\begin{tabular}{|c|c|c|c|c|c|c|}
\hline Results & \multicolumn{3}{|c|}{ Guidelines } & Sensor type & Maximal error & $\begin{array}{l}\text { Standard } \\
\text { deviation }\end{array}$ \\
\hline Fig. 3a (setup \#1) & & $\checkmark$ & & $\mathrm{CCD}$ & $294 \mathrm{~nm}$ & $\mathrm{x}$ \\
\hline Fig. 3c (setup \#1) & & $\checkmark$ & $\checkmark$ & $\mathrm{CCD}$ & $278 \mathrm{~nm}$ & $\mathrm{x}$ \\
\hline Fig. 4 (setup \#1) & $\checkmark$ & $\checkmark$ & $\checkmark$ & CCD & $22 \mathrm{~nm}$ & $5 \mathrm{~nm}$ \\
\hline Setup \#2 & $\checkmark$ & $\checkmark$ & $\checkmark$ & CMOS & $24 \mathrm{~nm}$ & $5 \mathrm{~nm}$ \\
\hline
\end{tabular}

TABLE I: Results sum-up showing the efficiency of the guidelines upon two different setups ("setup \#1" and "setup \#2").

factor was the warm-up of the camera which reduce the maximal error from $278 \mathrm{~nm}$ to $22 \mathrm{~nm}$, corresponding to a reduction of $92 \%$. These results show that, even within a short period of time, uncertainties can be high at very small scales without careful precautions and warm-ups.

To conclude, with the proposed guidelines, the position measurement uncertainty is estimated to $5 \mathrm{~nm}$. Moreover, the reproducibility standard deviation is $4 \mathrm{~nm}$ and the robustness against equipment-dependency has been demonstrated.

\section{REFERENCES}

[1] F. Fang, X. Zhang, W. Gao, Y. Guo, G. Byrne, and H. Hansen, "Nanomanufacturing-Perspective and applications," CIRP Annals, vol. 66, no. 2, pp. 683-705, 2017.

[2] D. Popa, R. Murthy, and A. Das, "M3 Deterministic, Multiscale, Multirobot Platform for Microsystems Packaging," IEEE Transactions on Automation Science and Engineering, vol. 6, no. 2, pp. 345-361, april 2009.

[3] H. C. Liaw and B. Shirinzadeh, "Robust generalised impedance control of piezo-actuated flexure-based four-bar mechanisms for micro/nano manipulation," Sensors and Actuators A: Physical, vol. 148, no. 2, pp. 443-453, december 2008.

[4] R. Wang and X. Zhang, "A planar 3-DOF nanopositioning platform with large magnification," Precision Engineering, vol. 46, pp. 221231, october 2016.

[5] D. Feng, M. Feng, E. Ozer, and Y. Fukuda, "A Vision-Based Sensor for Noncontact Structural Displacement Measurement," Sensors, vol. 15, no. 7, pp. 16557-16575, July 2015.

[6] V. Guelpa, P. Sandoz, M. A. Vergara, C. Clévy, N. Le Fort-Piat, and G. J. Laurent, "2D visual micro-position measurement based on intertwined twin-scale patterns," Sensors and Actuators A: Physical, vol. 248, pp. 272-280, September 2016.

[7] A. N. Andre, P. Sandoz, B. Mauze, M. Jacquot, and G. J. Laurent, "Sensing one nanometer over ten centimeters: A micro-encoded target for visual in-plane position measurement," IEEE/ASME Transactions on Mechatronics, pp. 1-1, 2020.

[8] S. Shirmohammadi and A. Ferrero, "Camera as the instrument: The rising trend of vision based measurement," IEEE Instrumentation \& Measurement Magazine, vol. 17, no. 3, pp. 41-47, june 2014.

[9] "International Vocabulary of Metrology - Basic and General Concepts and Associated Terms," Joint Committee for Guides in Metrology, Standard, 2012.

[10] C. Clévy, M. Rakotondrabe, and N. Chaillet, Eds., Signal Measurement and Estimation Techniques for Micro and Nanotechnology. New York: Springer Verlag, 2011.

[11] M. Greminger and B. Nelson, "Vision-based force measurement," IEEE Transactions on Pattern Analysis and Machine Intelligence, vol. 26, no. 3, pp. 290-298, march 2004.

[12] B. Pan, K. Qian, H. Xie, and A. Asundi, "Two-dimensional digital image correlation for in-plane displacement and strain measurement: A review," Measurement Science and Technology, vol. 20, no. 6, p. 062001 , june 2009.

[13] C.-S. HO, "Precision of Digital Vision Systems," IEEE transactions on pattern analysis and machine intelligence, p. 9, 1983.
[14] G. Luo, O. Chutatape, and H. Fang, "Experimental study on nonuniformity of line jitter in ccd images," Applied Optics, vol. 40, no. 26, pp. 4716-4720, 2001.

[15] D. J. Cappelleri, G. Piazza, and Vijay Kumar, "A two dimensional vision-based force sensor for microrobotic applications," Sensors and Actuators A: Physical, vol. 171, no. 2, pp. 340-351, november 2011.

[16] J. D. Skibicki and S. Judek, "Influence of vision measurement system spatial configuration on measurement uncertainty, based on the example of electric traction application," Measurement, vol. 116, pp. 281-298, february 2018.

[17] A. Belhaoua, S. Kohler, and E. Hirsch, "Error Evaluation in a Stereovision-Based 3D Reconstruction System," EURASIP Journal on Image and Video Processing, vol. 2010, pp. 1-12, 2010.

[18] C. Zhao, C. F. Cheung, and M. Liu, "Nanoscale measurement with pattern recognition of an ultra-precision diamond machined polar microstructure," Precision Engineering, november 2018

[19] J. Kokorian, F. Buja, and W. M. van Spengen, "In-Plane Displacement Detection With Picometer Accuracy on a Conventional Microscope," Journal of Microelectromechanical Systems, vol. 24, no. 3, pp. 618625 , june 2015.

[20] A. Ya'akobovitz, S. Krylov, and Y. Hanein, "Nanoscale displacement measurement of electrostatically actuated micro-devices using optical microscopy and digital image correlation," Sensors and Actuators A: Physical, vol. 162, no. 1, pp. 1-7, july 2010.

[21] C. Yamahata, E. Sarajlic, G. J. M. Krijnen, and M. A. M. Gijs, "Subnanometer Translation of Microelectromechanical Systems Measured by Discrete Fourier Analysis of CCD Images," Journal of Microelectromechanical Systems, vol. 19, no. 5, pp. 1273-1275, october 2010.

[22] V. Guelpa, G. J. Laurent, P. Sandoz, J. Galeano Zea, and C. Clévy, "Subpixelic measurement of large 1d displacements: Principle, processing algorithms, performances and software," Sensors, vol. 14, no. 3, pp. 5056-5073, 2014.

[23] N. Tan, C. Clévy, and N. Chaillet, "Calibration of single-axis nanopositioning cell subjected to thermal disturbance," in IEEE International Conference On Robotics and Automation, 2013, pp. 3660-3665.

[24] P. R. Muniz, R. de Araújo Kalid, S. P. N. Cani, and R. da Silva Magalhães, "Handy method to estimate uncertainty of temperature measurement by infrared thermography," Optical Engineering, vol. 53, no. 7, p. 074101, july 2014.

[25] N. Chaillet and S. Régnier, Eds., Microrobotics for Micromanipulation. London: ISTE, 2010, oCLC: 699766956.

[26] Y. Meng and H. Zhuang, "Autonomous robot calibration using vision technology," Robotics and Computer-Integrated Manufacturing, vol. 23, no. 4, pp. 436-446, august 2007.

[27] M. G. de Faria, Y. Haddab, Y. Le Gorrec, and P. Lutz, "Influence of mechanical noise inside a scanning electron microscope," Review of Scientific Instruments, vol. 86, no. 4, p. 045105, april 2015

[28] P. Sandoz, I. Elhechmi, and T. Gharbi, "Toward stereovisual monitoring of three-dimensional translations with submicrometer resolution," Journal of the Optical Society of America A, vol. 29, no. 11, p. 2451, november 2012

[29] H. Handel, "Analyzing the Influences of Camera Warm-Up Effects on Image Acquisition," Computer Vision - ACCV 2007, vol. 4844, p. 11, 2007. 